Note Added in Proof. When the reaction of 1 with $\mathrm{PhSCl}$ in a 1:3 molar ratio is carried out in the absence of a solvent, the yield of the diazene $2 \mathrm{e}$ is $\geqslant 90 \%$.

Acknowledgment. We thank the NSERC (Canada) for support in the form of operating and infrastructure grants, Mr. Glen Bigam (University of Alberta) for recording the ESR spectra, and Drs. A. Rauk and T. G. Back for helpful discussions. V.C. and M. N.S.R. thank IIT Kanpur and IIT Madras, respectively, for a sabbatical leave.

Registry No. 1, 24261-90-3; 2a, 136276-65-8; 2b, 136276-66-9; 2c, 136276-67-0; 2d, 136276-68-1; 2e, 136276-69-2; 3a, 136276-70-5; 3b,
136276-71-6; 3c, 136276-72-7; 4b, 136276-73-8; 4c, 136276-74-9; 5a, 86602-13-3; 5b, 136396-53-7; 6a, 136276-75-0; 6b, 136276-76-1; 6c 136276-77-2; $\mathrm{PhSCl}, 931-59-9 ; \mathrm{Cl}_{3} \mathrm{CSCl}, 594-42-3 ; 2,4-\left(\mathrm{NO}_{2}\right)_{2} \mathrm{C}_{6} \mathrm{H}_{3} \mathrm{SCl}$, 528-76-7; $\mathrm{Cl}_{3} \mathrm{SeMe}, 37826-07-6 ; \mathrm{TMS}-\mathrm{N}=\mathrm{C}\left[\mathrm{N}(\mathrm{TMS})_{2}{ }_{2} \mathrm{C}_{6} \mathrm{H}_{4}-\mathrm{p}-\mathrm{Me}\right.$, 117357-77-4; 2,4-( $\left.\mathrm{NO}_{2}\right)_{2} \mathrm{C}_{6} \mathrm{H}_{3} \mathrm{SN}=\mathrm{C}(\mathrm{Ph}) \mathrm{NH}_{2}, 136276-78-3$; TMS$\mathrm{N}=\mathrm{C}\left[\mathrm{N}(\mathrm{TMS})_{2}\right] \mathrm{C}_{6} \mathrm{H}_{4}-p-\mathrm{CF}_{3}, 117357-81-0 ; 2,4-\left(\mathrm{NO}_{2}\right)_{2} \mathrm{C}_{6} \mathrm{H}_{3} \mathrm{SN}=\mathrm{C}$ $(\mathrm{Ph}) \mathrm{N}=\mathrm{NC}(\mathrm{Ph})=\mathrm{NS}-2,4-\left(\mathrm{NO}_{2}\right)_{2} \mathrm{C}_{6} \mathrm{H}_{3}, 136276-79-4 ; \mathrm{PhSeCl}, 5707$ 04-0; $\mathrm{PhSeCl}_{3}, 42572-42-9$.

Supplementary Material Available: For $\mathbf{4 b}$, listings of non-hydrogen and hydrogen coordinates, anisotropic thermal parameters, bond distances, bond angles, torsion angles, and experimental details for the X-ray analyses ( 7 pages); listings of structure factor amplitudes ( 23 pages). Ordering information is given on any current masthead page.

Contribution from the Institut für Anorganische Chemie, Universität des Saarlandes, WD-6600 Saarbrücken, Germany

\title{
Halide-Complexing Characteristics of Germa- and Stannaimine Cages
}

\author{
Michael Veith, ${ }^{*}$ Joachim Fischer, Timothy R. Prout, Martin Nötzel, Peter Hobein, and Volker Huch
}

Received April 30, 1991

The cations $\mathrm{M}_{3}(\mathrm{~N}-t-\mathrm{Bu})_{4} \mathrm{H}_{3}{ }^{+}(\mathrm{M}=\mathrm{Ge}, \mathrm{Sn})$ coordinate very efficiently via hydrogen bridges to chloride, bromide, and iodide anions. These molecular complexes have the general formula $\mathrm{M}_{3}(\mathrm{~N}-t-\mathrm{Bu})_{4} \mathrm{H}_{3} \mathrm{X}(\mathrm{M}=\mathrm{Ge}, \mathrm{X}=\mathrm{Cl}$ (1), $\mathrm{Br}$ (2), I (3); $\mathrm{M}=\mathrm{Sn}, \mathrm{X}=\mathrm{Cl}$ (4), $\mathrm{Br}(5), \mathrm{I}(6))$ and are obtained by three different methods. The newly synthesized compounds have been thoroughly characterized by IR, NMR, and mass spectroscopy. X-ray crystal structure determinations of $1,3,4$, and 5 reveal the molecules to be built of a $\mathrm{M}_{3} \mathrm{~N}_{4}$-norcubane-type skeleton, which is attached by nitrogen-hydrogen bonds to a $\mathrm{H}_{3} \mathrm{X}$ trigonal pyramid. The overall closed cage " $\mathrm{M}_{3} \mathrm{~N}_{4} \mathrm{H}_{3} \mathrm{X}^{\prime}$ has an approximate $3 \mathrm{~m}\left(C_{3 v}\right)$ symmetry with one nitrogen and the halogen atom situated on the 3-fold axis. The compounds 3 and 4 crystallize isotypically with 0.5 benzene/molecule in the space group $R \overline{3}$ with $a=b=11.990$ (7) $\AA, c=34.40$ (1) $\AA, \alpha=\beta=90^{\circ}, \gamma=120^{\circ}, Z=6$, and $V=4283$ (2) $\AA^{3}$ and $a=b=11.720$ (8) $\AA, c=35.109$ (15) $\AA$, $\alpha=\beta=90^{\circ}, \gamma=120^{\circ}, Z=6$, and $V=4176(2) \AA^{3}$, respectively, with the final $R$ factors being 0.057 (1369 reflections) and 0.032 (1152 reflections). Compound 1 crystallizes in the monoclinic space group $P 2_{1} / n$ with $a=10.188(6) \AA, b=19.923$ (8) $\AA, c=12.099(7) \AA, \beta=91.54(5)^{\circ}, Z=4$, and $V=2455$ (1) $\AA^{3}$. A total of 2859 independent data were collected, giving a final $R$ factor of 0.035 . Compound 5 is orthorhombic (space group Pnma) with $a=21.471$ (9) $\AA, b=11.753$ (6) $\AA, c=10.476$ (5) $\AA, Z=4$, and $V=2644$ (1) $\AA^{3}$. Refinement of this structure, using 1921 independent reflections, gave an $R$ factor of 0.033 . The compounds can be described as being composed of a spherical lipophilic part $\left(\mathrm{M}_{3}(\mathrm{~N}-\mathrm{t}-\mathrm{Bu})_{4}\right)$ and a somewhat protruding lipophobic part $\left(\mathrm{H}_{3} \mathrm{X}\right.$ pyramid). These properties seem to account for the ease with which these cages exchange the halogen anions in metathesis reactions with simple salts like $\mathrm{NaBr}, \mathrm{NaI}$, and $\mathrm{TlCl}$.

\section{Introduction}

Of the molecular amino and imino cages of $\mathrm{Ge}(\mathrm{II}), \mathrm{Sn}(\mathrm{II})$ and $\mathrm{Pb}$ (II), ${ }^{1}$ obtained by aminolysis of bis(amino)germylenes, -stannylenes, and -plumbylenes, ${ }^{2}$ the compounds $\mathrm{Ge}_{3}(\mathrm{~N}-\mathrm{t}-\mathrm{Bu})_{4} \mathrm{H}_{2}$ and $\mathrm{Sn}_{3}(\mathrm{~N}-t-\mathrm{Bu})_{4} \mathrm{H}_{2}$ are peculiar in several respects. They form "open" polycycles (i.e. the skeleton of the molecules may be visualized as a cube with a missing corner, the seven remaining corners being alternately occupied by $\mathrm{Ge} / \mathrm{Sn}$ and $\mathrm{N}$ atoms; for other compounds of this type see also ref 3 ) and show bond fluctuation behavior as established by variable-temperature NMR studies. ${ }^{4}$ One of these movements is a mutual intramolecular displacement of the two hydrogen atoms with respect to the three nitrogen atoms in the "open" corner of the cube. Taking the crystallographic dimensions of $\mathrm{Sn}_{3}(\mathrm{~N}-t-\mathrm{Bu})_{4} \mathrm{H}_{2}$ into account, ${ }^{5}$ it seemed clear to us that by introduction of a simple proton this intramolecular displacement should be arrested, forming a cation $\mathrm{Sn}_{3}(\mathrm{~N}-t-\mathrm{Bu})_{4} \mathrm{H}_{3}{ }^{+}$. The whole charged entity should behave as an excellent counterion to simple anions, as the three hydrogen atoms are pointing rigidly in one direction (see formula A). Since macrocyclic counterions or cryptands for simple anions like chloride, bromide, and iodide are less commonly available than for cationic species, ${ }^{6-10}$ it seemed

(1) (a) Veith, M. Chem. Rev. 1990, 90, 3. (b) Veith, M. Angew. Chem. 1987, 99, 1; Angew. Chem., Int. Ed. Engl. 1987, 26, 1.

(2) Veith, M. Comments Inorg. Chem. 1985, 4, 179.

(3) Holmes, R. R. Acc. Chem. Res. 1989, 22, 190

(4) Veith, M. Phosphorous, Sulfur and Sillicon 1989, 4l, 195.

(5) Veith, M. Z. Naturforsch. 1980, 35B, 20.

(6) (a) Lehn, J. M. Angew. Chem. 1990, 102, 1347. (b) Hosseini, M. W. Blacker, A. J.; Lehn, J. M. J. Am. Chem. Soc. 1990, 112, 3896. (c) Schmidtchen, F. P. Nachr. Chem. Tech. Lab. 1988, 36, 8. (d) Hosseini, M. W.; Lehn, J. M. Helv. Chim. Acta 1988, 71, 749. (e) Lehn, J. M.; Sonveaux, E.; Willard, A. K. J. Am. Chem. Soc, 1987, 100,4914 (f) Pascal, R. A., Jr.; Spergel, J.; Van Engen, D. Tetrahedron Lett. 1986, 27, 4099. (g) Pierre, J. L.: Barat, P. Bull. Soc. Chim. Fr. 1983, II-367. (h) Lehn, J. M. Acc. Chem. Res. 1978, $11,49$.

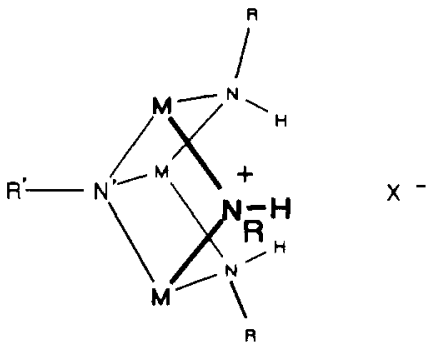

A

interesting to us to study the potential tridentate function of $\mathrm{Ge}_{3}(\mathrm{~N}-t-\mathrm{Bu})_{4} \mathrm{H}_{3}{ }^{+}$or $\mathrm{Sn}_{3}(\mathrm{~N}-t-\mathrm{Bu})_{4} \mathrm{H}_{3}{ }^{+}$toward these halogen anions. At the same time we intended to study the properties of the neutral complexes from a structural and chemical point of view.

\section{Experimental Section}

Apparatus and Materials. All operations were carried out under an inert atmosphere of nitrogen by using a modified Stock vacuum appa. ratus and Schlenk techniques. Infrared, ' $H$ NMR, and mass spectra were obtained by using Perkin-Elmer IR 883, Bruker WP 80 ( $80 \mathrm{MHz}$ ), and Finnigan MAT 90 (DCI ${ }^{+}$using isobutane) spectrometers, respectively. ${ }^{\prime} \mathrm{H}$ NMR chemical shifts as $\delta$ values were measured relative to internal $\mathrm{Me}_{4} \mathrm{Si}$. Elemental analyses were performed by Beller, Mi-

(7) Dietrich, B.; Guilhem, J.; Lehn, J. M.; Pascard, C.; Sonveaux, E. Helv. Chim. Acta 1984, 67, 91 .

(8) Graf, E.; Lehn, J. M. J. Am. Chem. Soc. 1976, 98, 6403.

(9) Metz, B.; Rosalky, J. M.; Weiss, R. J. Chem. Soc., Chem. Commun. 1976, 533.

(10) (a) Park, C. H.; Simmons, H. E. J. Am. Chem. Soc. 1968, 90, 2429. (b) Bell, R. A.; Christoph, G. G.; Fronzek, F. R.; Marsh, R. E. Science 1975, 190,15i. 
Table I. Crystallographic Data for $1,3.0 .5 \mathrm{C}_{6} \mathrm{H}_{6}, 40.5 \mathrm{C}_{6} \mathrm{H}_{6}$, and 5

\begin{tabular}{|c|c|c|c|c|}
\hline & 1 & $3 \cdot 0.5 \mathrm{C}_{6} \mathrm{H}_{6}$ & $4.0 .5 \mathrm{C}_{6} \mathrm{H}_{6}$ & 5 \\
\hline $\begin{array}{l}\text { mol formula } \\
\mathrm{fw}\end{array}$ & $\begin{array}{l}\mathrm{C}_{16} \mathrm{H}_{39} \mathrm{ClGe}_{3} \mathrm{~N}_{4} \\
540.79\end{array}$ & $\begin{array}{l}\mathrm{C}_{19} \mathrm{H}_{42} \mathrm{Ge}_{3} \mathrm{IN}_{4} \\
671.31\end{array}$ & $\begin{array}{l}\mathrm{C}_{19} \mathrm{H}_{42} \mathrm{ClN}_{4} \mathrm{Sn}_{3} \\
718.10\end{array}$ & $\begin{array}{l}\mathrm{C}_{16} \mathrm{H}_{39} \mathrm{BrN}_{4} \mathrm{Sn}_{3} \\
723.49\end{array}$ \\
\hline cryst dimens, mm & $0.35 \times 0.4 \times 0.4$ & $0.4 \times 0.4 \times 0.4$ & $0.2 \times 0.2 \times 0.4$ & $0.3 \times 0.4 \times 0.25$ \\
\hline $\begin{array}{l}\text { cryst color } \\
\text { cryst syst }\end{array}$ & $\begin{array}{l}\text { colorless } \\
\text { monoclinic }\end{array}$ & $\begin{array}{l}\text { colorless } \\
\text { rhombohedral }\end{array}$ & $\begin{array}{l}\text { colorless } \\
\text { rhombohedral }\end{array}$ & $\begin{array}{l}\text { light yellow } \\
\text { orthorhombic }\end{array}$ \\
\hline space group & $P 2_{1} / n$ & $R^{\overline{3}}$ & $R^{\overline{3}}$ & Pnma \\
\hline$a, \AA$ & $10.188(6)$ & $11.990(7)$ & $11.720(8)$ & $21.471(9)$ \\
\hline$\vec{b}, \AA$ & $19.923(8)$ & $11.990(7)$ & $11.720(8)$ & $11.753(6)$ \\
\hline$c, \AA$ & $12.099(7)$ & 34.40 (1) & $35.109(15)$ & $10.476(5)$ \\
\hline$\beta$ or $\gamma, \operatorname{deg}$ & $91.54(5)$ & 120.0 & 120.0 & 90.0 \\
\hline$V, \AA^{3}$ & 2455 (1) & $4283(2)$ & $4176(2)$ & $2644(1)$ \\
\hline$Z$ & 4 & 6 & 6 & 4 \\
\hline$\delta, \mathrm{g} \mathrm{cm}^{-3}$ & 1.463 & 1.56 & 1.71 & 1.818 \\
\hline no. of indep data & 2859 & 1369 & 1152 & 1921 \\
\hline no. of data with $F_{0}>n \sigma$ & $2666(n=3)$ & $965(n=2)$ & $966(n=2)$ & $1723(n=3)$ \\
\hline no. of variables & 260 & 73 & 105 & 158 \\
\hline$\mu, \mathrm{cm}^{-1}$ & 36.92 & 40.69 & 25.71 & 40.93 \\
\hline$R\left(F_{0}\right)$ & 0.035 & 0.057 & 0.032 & 0.0334 \\
\hline$R_{w}\left(F_{0}\right)$ & 0.037 & 0.057 & 0.031 & 0.0374 \\
\hline
\end{tabular}

kroanalytisches Laboratorium, Theaterstr. 23, Göttingen, Germany. The compounds $\mathrm{Me}_{2} \mathrm{Si}(t-\mathrm{BuN}), \mathrm{Ge}^{11} \mathrm{Me}_{2} \mathrm{Si}(t-\mathrm{BuN})_{2} \mathrm{Sn}^{12} \mathrm{Me}_{2} \mathrm{Si}(t-\mathrm{BuN})(t-$ $\mathrm{BuNH}) \mathrm{GeX}(\mathrm{X}=\mathrm{Cl}, \mathrm{Br}),{ }^{13} \mathrm{Me}_{2} \mathrm{Si}(t-\mathrm{BuN})(t-\mathrm{BuNH}) \mathrm{SnX}(\mathrm{X}=\mathrm{Cl}, \mathrm{Br}$, I), ${ }^{14} \mathrm{Ge}_{2}(t \text {-BuNH })_{2} \mathrm{Cl}_{2},{ }^{13} \mathrm{Sn}_{2}(t-\mathrm{BuNH})_{2} \mathrm{X}_{2}(\mathrm{X}=\mathrm{Cl}, \mathrm{Br}),{ }^{14} \mathrm{Ge}_{3}(\mathrm{~N}-t$ $\mathrm{Bu})_{4} \mathrm{H}_{2},{ }^{11}$ and $\mathrm{Sn}_{3}(\mathrm{~N}-\mathrm{t}-\mathrm{Bu})_{4} \mathrm{H}_{2}{ }^{15}$ were prepared as previously described. tert-Butylamine (Merck) was dried over $\mathrm{CaH}_{2}$ and distilled. $\mathrm{HCl}(\mathrm{g})$ and $\mathrm{HBr}(\mathrm{g})$ (Messer, Griesheim) were dried by using previously published methods ${ }^{16}$ and subsequently bubbled through toluene or benzene. The molarities of these solutions were determined by titration with both $\mathrm{NaOH}$ and $\mathrm{AgNO}_{3}{ }^{17}$ The solvents benzene, diethyl ether, and toluene were dried with $\mathrm{LiAlH}_{4}$ and/or sodium-benzophenone, distilled, and stored over $\mathrm{Na}$ wire. $\mathrm{NaBr}, \mathrm{NaI}$, and $\mathrm{TlCl}$ (Merck) were recrystallized and dried by published methods. ${ }^{16}$

The single crystals obtained were transferred to glass capillaries of appropriate sizes $(0.1-0.5-\mathrm{mm}$ diameter) and sealed under nitrogen. Crystal quality was examined by using either a Weissenberg camera (Stoe; Darmstadt, Germany) or a Buerger precession camera (Huber; Rimsting, Germany). The capillaries were then transferred to an automatic four-circle diffractometer (Siemens, type AED2) where data were collected by using the Wyckoff- $\omega$-scan technique with Mo K $\alpha$ radiation. Space group determinations and measurements of the cell constants were calculated on the basis of the photographs and then checked and refined again on the diffractometer. The details of data collections and structure determinations are summarized in Table I. All structures were solved by using standard Patterson techniques. Extinction corrections were not applied; however, the intensities were corrected for $L p$ effects. The absorption effects were taken into account by using reflections from a complete $\psi$ scan (empirical absorption correction). The hydrogen atoms on the methyl groups were treated together with the central carbon as a rigid group with tetrahedral local symmetry and standard bond lengths $(\mathrm{C}-\mathrm{H}=1.08 \AA)$. Final atomic positional parameters as well as the isotropic $B$ values calculated from $U_{b j}$ values are given in Table II. The most significant bond lengths and angles are collected in Table III. Anisotropic thermal parameters (Table SI), a full table of bond lengths and angles (Table SII), and a listing of observed and calculated structure factors are available as supplementary material. For computation a VAX-II computer was used with the programs SHELX, ${ }^{18}$ ORTEP, ${ }^{19}$ and SCHAKAL. ${ }^{20}$

Formation of $\mathrm{M}_{3}(\mathrm{~N}-\mathrm{t}-\mathrm{Bu})_{4} \mathrm{H}_{3} \mathrm{X}$. (A) $\mathrm{M}_{3}(\mathrm{~N}-\mathrm{t}-\mathrm{Bu})_{4} \mathrm{H}_{3}$ with $\mathrm{HX} /$ Benzene. To a stirred, dilute benzene solution $(10 \mathrm{~mL})$ of $\mathrm{M}_{3}(\mathrm{~N}-t-$ $\mathrm{Bu})_{4} \mathrm{H}_{2}(\mathrm{M}=\mathrm{Ge}, \mathrm{Sn})(1 \mathrm{mmol})$ was added an $\mathrm{HX} /$ benzene solution $(\mathrm{X}$

(11) Veith, M.; Grosser, M. Z. Naturforsch. 1982, 37B, 1375.

(12) Veith, M. Angew. Chem. 1975, 87, 287; Angew. Chem., Int. Ed. Engl. $1975,14,263$.

(13) Veith. M.: Hobein, P.: Rösler, R. Z. Naturforsch. 1989, 44B, 1067.

(14) Veith, M.; Jarczyk, M.; Huch, V. Chem. Ber. 1988, 121, 347.

(15) Veith, M.; Sommer, M.-L.; Jăger, D. Chem. Ber. 1979, 112, 2581

(16) Perrin, D. D.; Armarego, W. L. F. Purification of Laboratory Chemicals, 3rd ed.; Pergamon Press: Oxford, England, 1988.

(17) Jander, G.; Blasius, E. Einführung in das anorganisch-chemische Praktikum; S. Hirzel Verlag: Stuttgart, Germany, 1980.

(18) Sheldrick, G. M. SHELX-76/86, Program for Crystal Structure Determination: University of Cambridge: Cambridge, U.K., 1976.

(19) Johnson, C. K. ORTEP-II; Report ORNL-3794; Oak Ridge National Laboratory: Oak Ridge, TN, 1971.

(20) Keller, E. SCHAKAL-88, Programm zur Darstellung von Kristallstrukturen; Universităt Freiburg: Freiburg, Germany, 1988.
$=\mathrm{Cl}, \mathrm{Br}$ ), e.g. $9.1 \mathrm{~mL}$ of $0.11 \mathrm{M}$ benzene $/ \mathrm{HCl}$, dropwise over $2 \mathrm{~h}$. As the solution was added, $1,2,4$, and 5 precipitated as white solids. After $2 \mathrm{~h}$ of stirring, the volatiles were removed in vacuo resulting in 98-99\% analytically pure 0.5 benzene adducts of $1,2,4$, and 5 . The physical and spectroscopic data of 1-6 are collected in Table IV (see below for formation of 3 and 6 ).

(B) $\mathrm{Me}_{2} \mathrm{Si}(\mathrm{N}-t-\mathrm{Bu})_{2} \mathrm{M}$ and $\mathrm{Me}_{2} \mathrm{Si}(t-\mathrm{BuNH})(\mathrm{N}-t-\mathrm{Bu}) \mathrm{MX}$ with $t$ BuNH $_{2}$. tert-Butylamine $(1.26 \mathrm{~mL}, 12 \mathrm{mmol})$ was added dropwise to a stirred mixture of $\mathrm{Me}_{2} \mathrm{Si}(\mathrm{N}-t-\mathrm{Bu})_{2} \mathrm{Ge}$ or $\mathrm{Me}_{2} \mathrm{Si}(\mathrm{N}-t-\mathrm{Bu})_{2} \mathrm{Sn}$ (3 mmol) and $\mathrm{Me}_{2} \mathrm{Si}(t-\mathrm{BuNH})(\mathrm{N}-t-\mathrm{Bu}) \mathrm{GeCl}$ or $\mathrm{Me}_{2} \mathrm{Si}(t-\mathrm{BuNH})(\mathrm{N}-t-\mathrm{Bu}) \mathrm{SnX}(\mathrm{X}$ $=\mathrm{Cl}, \mathrm{Br}, \mathrm{I})(6 \mathrm{mmol})$ in benzene $(5 \mathrm{~mL})$. The yellow or orange solution changed to colorless or slightly yellow shortly after addition of the amine as a crystalline solid precipitated. The solution was refluxed for $2 \mathrm{~h}$ in order to ensure completion of the reaction. The volatiles were removed under vacuum resulting in colorless crystalline solids. Recrystallization from benzene resulted in pure products: $1 \cdot 0.5 \mathrm{C}_{6} \mathrm{H}_{6}(90 \%), 40.5 \mathrm{C}_{6} \mathrm{H}_{6}$ $(87 \%), 5 \cdot 0.5 \mathrm{C}_{6} \mathrm{H}_{6}(95 \%)$, and $6.0 .5 \mathrm{C}_{6} \mathrm{H}_{6}(94 \%)$. When 1 and 5 were recrystallized from toluene, solvent-free crystals were obtained $(91 \%)$.

(C) $\mathrm{Me}_{2} \mathrm{Si}(\mathrm{N}-t-\mathrm{Bu})_{2} \mathrm{M}$ and $[(t-\mathrm{BuNH}) \mathrm{MX}]_{2}$ with $t-\mathrm{BuNH}_{2}$. tert-Butylamine $(0.95 \mathrm{~mL}, 9 \mathrm{mmol})$ was added dropwise to a mixture of $\mathrm{Me}_{2} \mathrm{Si}(\mathrm{N}-t-\mathrm{Bu})_{2} \mathrm{M}(6.0 \mathrm{mmol})$ and $[(t-\mathrm{BuNH}) \mathrm{GeCl}]_{2}$ or $[(t-\mathrm{BuNH})-$ $\mathrm{SnX}]_{2}(\mathrm{X}=\mathrm{Cl}, \mathrm{Br}, \mathrm{I})(1.5 \mathrm{mmol})$ in benzene $(3 \mathrm{~mL})$. Again the orange color of the solution changed to a light yellow with addition of the amine and a colorless crystalline solid precipitated. The solution was refluxed for $2 \mathrm{~h}$. All volatiles were removed in vacuo and the solids recrystallized from benzene ( $5 \mathrm{~mL}): 1 \cdot 0.5 \mathrm{C}_{6} \mathrm{H}_{6}(88 \%), 4 \cdot 0.5 \mathrm{C}_{6} \mathrm{H}_{6}(92 \%), 5 \cdot 0.5 \mathrm{C}_{6} \mathrm{H}_{6}$ (93\%), 6.0.5 $\mathrm{C}_{6} \mathrm{H}_{6}(93 \%)$.

For physical and spectroscopic data of $1,2,4,5$, and 6, see Table IV Anal. Calc for $\mathrm{C}_{16} \mathrm{H}_{39} \mathrm{ClGe}_{3} \mathrm{~N}_{4}, M_{\mathrm{r}}=540.79(1): \mathrm{C}, 35.54 ; \mathrm{H}, 7.27$; $\mathrm{Cl}, 6.56 ; \mathrm{N}, 10.36$. Found: $\mathrm{C}, 35.51 ; \mathrm{H}, 7.22 ; \mathrm{Cl}, 6.62 ; \mathrm{N}, 10.23$. Calc for $\mathrm{C}_{19} \mathrm{H}_{42} \mathrm{ClGe}_{3} \mathrm{~N}_{4}, M_{\mathrm{r}}=579.80\left(1 \cdot 0.5 \mathrm{C}_{6} \mathrm{H}_{6}\right): \mathrm{C}, 39.36 ; \mathrm{H}, 7.30 ; \mathrm{Cl}$, $6.11 ; \mathrm{N}, 9.66$. Found: $\mathrm{C}, 40.23 ; \mathrm{H}, 7.41 ; \mathrm{Cl}, 6.01 ; \mathrm{N}, 9.76$. Calc for $\mathrm{C}_{16} \mathrm{H}_{39} \mathrm{BrGe}_{3} \mathrm{~N}_{4}, M_{\mathrm{r}}=585.25(2): \mathrm{C}, 32.84 ; \mathrm{H}, 6.72 ; \mathrm{N}, 9.57$. Found: C, 33.54; H, 6.78; N, 9.42. Calc for $\mathrm{C}_{19} \mathrm{H}_{42} \mathrm{ClN}_{4} \mathrm{Sn}_{3}, M_{\mathrm{r}}=718.10$ $\left(4.0 .5 \mathrm{C}_{6} \mathrm{H}_{6}\right): C, 31.78 ; \mathrm{H}, 5.90 ; \mathrm{Cl}, 4.94 ; \mathrm{N}, 7.80$. Found: $\mathrm{C}, 32.07 ; \mathrm{H}$, $5.45 ; \mathrm{Cl}, 4.86 ; \mathrm{N}, 7.66$. Calc for $\mathrm{C}_{19} \mathrm{H}_{42} \mathrm{BrN}_{4} \mathrm{Sn}_{3}, M_{\mathrm{r}}=762.55$ (5. $\left.0.5 \mathrm{C}_{6} \mathrm{H}_{6}\right): \mathrm{C}, 29.93 ; \mathrm{H}, 5.50 ; \mathrm{Br}, 10.48 ; \mathrm{N}, 7.35$. Found: $\mathrm{C}, 30.13 ; \mathrm{H}$, $5.55 ; \mathrm{Br}, 10.00 ; \mathrm{N}, 7.41$. Calc for $\mathrm{C}_{19} \mathrm{H}_{42} \mathrm{IN}_{4} \mathrm{Sn}_{3}, M_{\mathrm{r}}=809.54(6$ $\left.0.5 \mathrm{C}_{6} \mathrm{H}_{6}\right): \mathrm{C}, 28.19 ; \mathrm{H}, 5.23 ; \mathrm{I}, 15.68 ; \mathrm{N}, 6.92$. Found: $\mathrm{C}, 28.46 ; \mathrm{H}$, $5.20 ; \mathrm{I}, 15.46 ; \mathrm{N}, 6.97$.

Formation of $\left[(t-\mathrm{BuNH}) \mathrm{SnI}_{2}\right.$ (7). Me $\mathrm{Si}(t-\mathrm{BuNH})(\mathrm{N}-t-\mathrm{Bu}) \mathrm{SnI}$ with $t$-BuNH . To a stirred solution of $\mathrm{Me}_{2} \mathrm{Si}(t-\mathrm{BuNH})(\mathrm{N}-t-\mathrm{Bu}) \mathrm{SnI}(1.430$ g. $3.2 \mathrm{mmol}$ ) was added tert-butylamine $(0.34 \mathrm{~mL}, 3.2 \mathrm{mmol})$ dropwise. After 2 days of stirring at room temperature all volatiles were removed in vacuo producing a slightly yellow microcrystalline solid of [ $(t-$ BuNH)SnI ] 2 (7): yield $1.23 \mathrm{~g}, 89 \%$; mp $124^{\circ} \mathrm{C}$. Anal. Calc for $\mathrm{C}_{8} \mathrm{H}_{20} \mathrm{I}_{2} \mathrm{~N}_{2} \mathrm{Sn}_{2}, M_{\mathrm{r}}=635.48:$ C, $15.12 ; \mathrm{H}, 3.17 ; \mathrm{I}, 39.94 ; \mathrm{N}, 4.41$. Found: $\mathrm{C}, 14.89 ; \mathrm{H}, 3.38 ; \mathrm{I}, 39.45 ; \mathrm{N}, 4.47$. ' $1 \mathrm{H} \mathrm{NMR}\left(\mathrm{C}_{6} \mathrm{D}_{6}\right): \delta 0.91$ [s, $\left.18 \mathrm{H}, \mathrm{C}\left(\mathrm{CH}_{3}\right)_{3}\right]$. Molecular mass: calc, $635.48 \mathrm{~g} / \mathrm{mol}$; found (by cryoscopy in benzene), $653.8 \mathrm{~g} / \mathrm{mol}$.

Anion-Exchange Reactions. (A) (N-t-Bu) $(t-\mathrm{BuNH})_{3} \mathrm{M}_{3} \mathrm{Cl}$ with $\mathrm{NaX}$ $(X=B r, I)$. In a typical experiment, a $0.02 \mathrm{M}$ solution of 1 or 4 in benzene or toluene was added to an equimolar amount of the crystalline salt. The suspension was vigorously stirred and periodically monitored by ${ }^{1} H$ NMR spectroscopy. Upon completion of the reaction the crystalline solid was separated by filtration and was identified as $\mathrm{NaCl}$ by $X$-ray powder diffraction. The filtrate was concentrated in vacuo and 
Table II. Atomic Positional Parameters and Isotropic $B$ Values $\left(\AA^{2}\right)$ (Calculated from $U_{1 j}$ ) of $1,3.0 .5 \mathrm{C}_{6} \mathrm{H}_{6}, 4 \cdot 0.5 \mathrm{C}_{6} \mathrm{H}_{6}$, and 5

\begin{tabular}{|c|c|c|c|c|}
\hline atom & $x$ & $y$ & $z$ & $B$ \\
\hline $\begin{array}{l}\mathrm{Ge}(1) \\
\mathrm{Ge}(2) \\
\mathrm{Ge}(3) \\
\mathrm{Cl} \\
\mathrm{N}(1) \\
\mathrm{N}(2) \\
\mathrm{H}(1) \\
\mathrm{N}(3) \\
\mathrm{H}(2) \\
\mathrm{N}(4) \\
\mathrm{H}(3) \\
\mathrm{C}(1) \\
\mathrm{C}(11) \\
\mathrm{C}(12) \\
\mathrm{C}(13) \\
\mathrm{C}(2) \\
\mathrm{C}(21) \\
\mathrm{C}(22) \\
\mathrm{C}(23) \\
\mathrm{C}(3) \\
\mathrm{C}(31) \\
\mathrm{C}(32) \\
\mathrm{C}(33) \\
\mathrm{C}(4) \\
\mathrm{C}(41) \\
\mathrm{C}(42) \\
\mathrm{C}(43)\end{array}$ & $\begin{array}{l}0.83746(5) \\
0.59245(5) \\
0.58944(5) \\
0.5630(2) \\
0.6990(4) \\
0.7243(4) \\
0.691(5) \\
0.4872(3) \\
0.5056(0) \\
0.7199(4) \\
0.6712(0) \\
0.7399(5) \\
0.8303(7) \\
0.6193(6) \\
0.8137(7) \\
0.7904(5) \\
0.6851(6) \\
0.8832(6) \\
0.8638(6) \\
0.3398(5) \\
0.3054(6) \\
0.2870(6) \\
0.2873(6) \\
0.7840(5) \\
0.6755(6) \\
0.8803(7) \\
0.8514(7)\end{array}$ & $\begin{array}{c}1 \\
1 \\
0.09487(3) \\
0.07038(2) \\
0.04807(3) \\
0.25813(6) \\
0.0237(2) \\
0.1450(2) \\
0.173(2) \\
0.1015(2) \\
0.1471(0) \\
0.1245(2) \\
0.1696(0) \\
-0.0475(3) \\
-0.0531(3) \\
-0.0913(3) \\
-0.0715(3) \\
0.1748(3) \\
0.2052(3) \\
0.2301(3) \\
0.1200(3) \\
0.0937(3) \\
0.0213(3) \\
0.1123(4) \\
0.1412(3) \\
0.1370(3) \\
0.1554(4) \\
0.1941(4) \\
0.0740(3)\end{array}$ & $\begin{array}{r}0.25669(4) \\
0.11631(4) \\
0.36332(4) \\
0.2879(1) \\
0.2351(3) \\
0.1447(3) \\
0.172(3) \\
0.2467(3) \\
0.2648(0) \\
0.3802(3) \\
0.3621(0) \\
0.2206(4) \\
0.1227(5) \\
0.1992(5) \\
0.3244(5) \\
0.0466(4) \\
-0.0308(5) \\
0.0844(5) \\
-0.0133(5) \\
0.2404(4) \\
0.2121(5) \\
0.3535(5) \\
0.1521(5) \\
0.4926(4) \\
0.5723(5) \\
0.4827(5) \\
0.5312(5)\end{array}$ & $\begin{array}{l}3.46(3) \\
2.99(3) \\
3.32(3) \\
4.82(7) \\
2.9(2) \\
3.3(2) \\
2(1) \\
3.0(2) \\
2.5(9) \\
3.4(2) \\
5(1) \\
3.8(2) \\
5.4(3) \\
4.9(3) \\
5.8(3) \\
4.0(2) \\
5.9(3) \\
5.7(3) \\
5.5(3) \\
3.9(2) \\
5.1(3) \\
5.7(3) \\
5.5(3) \\
4.2(3) \\
6.0(3) \\
6.4(4) \\
5.9(3)\end{array}$ \\
\hline $\begin{array}{l}\mathrm{I} \\
\mathrm{Ge} \\
\mathrm{N}(1) \\
\mathrm{N}(2) \\
\mathrm{C}(1) \\
\mathrm{C}(2) \\
\mathrm{C}(3) \\
\mathrm{C}(4) \\
\mathrm{C}(5) \\
\mathrm{C}(6) \\
\mathrm{C}(7) \\
\mathrm{C}(8)\end{array}$ & $\begin{array}{c}0.0000(0) \\
0.0841(1) \\
0.0000(0) \\
0.1573(8) \\
0.0000(0) \\
-0.070(1) \\
0.302(1) \\
0.336(1) \\
0.335(1) \\
0.371(1) \\
0.013(5) \\
0.207(4)\end{array}$ & $\begin{array}{c}3.0 .5 \mathrm{C}_{6} \mathrm{H}_{6} \\
0.0000(0) \\
0.1683(1) \\
0.0000(0) \\
0.0785(9) \\
0.0000(0) \\
-0.139(1) \\
0.150(1) \\
0.062(1) \\
0.272(1) \\
0.185(1) \\
0.123(3) \\
0.103(4)\end{array}$ & $\begin{array}{l}0.16486(3) \\
0.29224(3) \\
0.3209(3) \\
0.2601(2) \\
0.3643(4) \\
0.3796(5) \\
0.2525(3) \\
0.2289(4) \\
0.2289(4) \\
0.2918(4) \\
0.5011(8) \\
0.503(1)\end{array}$ & $\begin{array}{l}7.16(9) \\
6.5(1) \\
6.5(8) \\
6.0(8) \\
7(1) \\
9.0(3) \\
7(1) \\
8.6(3) \\
8.6(3) \\
8.9(4) \\
15.3(8) \\
2.3(8)\end{array}$ \\
\hline $\begin{array}{l}\text { Sn } \\
C l \\
N(1) \\
N(2) \\
C(1) \\
C(11) \\
C(12) \\
C(13) \\
C(2) \\
C(21) \\
C(3) \\
H(1) \\
H(2)\end{array}$ & $\begin{array}{c}-0.09523(3) \\
0.0000(0) \\
0.0886(4) \\
0.0000(0) \\
0.1606(6) \\
0.0694(7) \\
0.2812(7) \\
0.2006(8) \\
0.0000(0) \\
0.0709(6) \\
-0.599(1) \\
0.068(5) \\
-0.224(8)\end{array}$ & $\begin{array}{c}4 \cdot 0.5 \mathrm{C}_{6} \mathrm{H}_{6} \\
-0.19054(3) \\
0.0000(0) \\
-0.0879(4) \\
0.0000(0) \\
-0.1596(6) \\
-0.2818(6) \\
-0.0688(7) \\
-0.1988(8) \\
0.0000(0) \\
-0.0689(6) \\
-0.1997(8) \\
-0.059(5) \\
-0.105(8)\end{array}$ & $\begin{array}{l}0.21101(1) \\
0.32469(8) \\
0.2452(1) \\
0.1814(2) \\
0.2548(2) \\
0.2776(2) \\
0.2777(2) \\
0.2177(2) \\
0.1388(3) \\
0.1236(2) \\
-0.3337(3) \\
0.269(2) \\
0.003(3)\end{array}$ & $\begin{array}{l}3.01(4) \\
4.6(1) \\
3.0(3) \\
2.8(3) \\
4.1(5) \\
5.2(5) \\
5.7(6) \\
5.8(6) \\
2.9(4) \\
4.3(5) \\
7.0(7) \\
3(1) \\
9(2)\end{array}$ \\
\hline $\begin{array}{l}\operatorname{Sn}(1) \\
\operatorname{Sn}(2) \\
\mathrm{Br} \\
N(1) \\
H(1) \\
N(2) \\
H(2) \\
N(3) \\
C(1) \\
C(11) \\
C(12) \\
C(13) \\
C(2) \\
C(21) \\
C(22) \\
C(3) \\
C(31) \\
C(32)\end{array}$ & $\begin{array}{l}0.43441(2) \\
0.40438(3) \\
0.23506(5) \\
0.3600(2) \\
0.325(3) \\
0.3902(3) \\
0.349(4) \\
0.4713(3) \\
0.3381(3) \\
0.3110(4) \\
0.2877(5) \\
0.3911(4) \\
0.3948(5) \\
0.4653(6) \\
0.3632(5) \\
0.5404(5) \\
0.5806(5) \\
0.5562(4)\end{array}$ & $\begin{array}{c}5 \\
0.10678(4) \\
0.2500(0) \\
0.2500(0) \\
0.1199(4) \\
0.156(5) \\
0.2500(0) \\
0.2500(0) \\
0.2500(0) \\
0.0133(6) \\
-0.0663(7) \\
0.0419(8) \\
-0.0447(8) \\
0.2500(0) \\
0.2500(0) \\
0.3563(9) \\
0.2500(0) \\
0.2500(0) \\
0.352(1)\end{array}$ & $\begin{array}{l}0.90635(4) \\
0.63787(6) \\
0.9154(1) \\
0.7610(5) \\
0.800(5) \\
1.0111(7) \\
0.986(8) \\
0.7969(7) \\
0.6980(7) \\
0.7962(9) \\
0.6007(8) \\
0.631(1) \\
1.154(1) \\
1.193(1) \\
1.2074(9) \\
0.763(1) \\
0.885(1) \\
0.689(1)\end{array}$ & $\begin{array}{l}3.73(3) \\
4.01(3) \\
4.69(5) \\
3.3(2) \\
3(1) \\
3.2(3) \\
2(2) \\
3.7(4) \\
4.5(3) \\
6.5(5) \\
6.5(5) \\
7.9(6) \\
5.2(6) \\
6.3(7) \\
8.4(6) \\
5.4(6) \\
7.0(8) \\
8.0(6)\end{array}$ \\
\hline
\end{tabular}

the crystalline material spectroscopically $\left({ }^{1} \mathrm{H}\right.$ NMR, IR, MS) characterized. In the case of $2(98 \%)$ and $3(98 \%)$ elemental analyses were performed. Further data for $\mathbf{2}$ and $\mathbf{3}$ are found in Table IV.

(B) $t$-BuN $(t-B u N H)_{3} M_{3} I$ with TKCl. These reactions were carried out similarly to method $\mathrm{A}$ above. In these reactions $\mathrm{I}^{-}$exchanged with $\mathrm{Cl}^{-}$ forming TII and the compounds 1 and 4, respectively (yield $98 \%$, characterized by ${ }^{1} \mathrm{H}$ NMR and IR). Anal. Calc for $\mathrm{C}_{19} \mathrm{H}_{42} 1 \mathrm{IGe}_{3} \mathrm{~N}_{4}, M_{\mathrm{f}}=$ $671.31\left(3.0 .5 \mathrm{C}_{6} \mathrm{H}_{6}\right)$ : C, 33.99; $\mathrm{H}, 6.31 ; \mathrm{N}, 8.35$. Found: $\mathrm{C}, 33.31 ; \mathrm{H}$, 6.34: N, 8.44.

Reaction of $\mathrm{Sn}_{3}(\mathrm{~N}-t-\mathrm{Bu})_{4} \mathrm{H}_{2}(10)$ with Excess $\mathrm{HCl} . \mathrm{Sn}_{3}(\mathrm{~N}-t-\mathrm{Bu})_{4} \mathrm{H}_{2}$ $(2 \mathrm{mmol})$ in benzene $(10 \mathrm{~mL})$ was treated with an $\mathrm{HCl} /$ benzene solution in molar portions. The first molar equivalent produced 4 . As excess $\mathrm{HCl}$ was added $\left(\mathrm{Sn}_{3}(\mathrm{~N}-t-\mathrm{Bu})_{4} \mathrm{H}_{2}: \mathrm{HCl}=1: 6\right), \mathrm{SnCl}_{2} \cdot \mathrm{H}_{2} \mathrm{~N}-t$ - Bu was obtained: yield $1.18 \mathrm{~g}, 75 \%$. The elemental analysis and a singlet in the ${ }^{1} \mathrm{H}$ NMR spectrum at $\delta 0.73$ are consistent with those previously reported. ${ }^{14}$ After further addition of $\mathrm{HCl}\left(\mathrm{Sn}_{3}(\mathrm{~N}-t-\mathrm{Bu})_{4} \mathrm{H}_{2}: \mathrm{HCl}=1: 9\right), t-\mathrm{BuNH}_{3}{ }^{+} \mathrm{SnCl}_{3}^{-}$ was obtained: yield $1.6 \mathrm{~g}, 89 \%$. This product was characterized by ${ }^{\prime} \mathrm{H}$ NMR spectroscopy $\left[\delta 1.33\left(\mathrm{CD}_{3} \mathrm{CN}\right)\right]$ and its melting point $\left(135^{\circ} \mathrm{C}\right) .^{14}$

\section{Results and Discussion}

Syntheses. As discussed in the Introduction the iminogermylene and -stannylenes $(\mathrm{N}-t-\mathrm{Bu})_{2}(t-\mathrm{BuNH})_{2} \mathrm{Ge}_{3}{ }^{11}$ and $(\mathrm{N}-t-\mathrm{Bu})_{2}(t-$ $\mathrm{BuNH})_{2} \mathrm{Sn}_{3}{ }^{15}$ react quantitatively with dilute $\mathrm{HX}$ solutions in benzene forming $t$ - $\mathrm{BuN}(t-\mathrm{BuNH})_{3} \mathrm{M}_{3} \mathrm{X}(1,2,4,5)$ (eq 1$)$. Other

$$
\begin{aligned}
M_{3}(\mathrm{~N} / \mathrm{Bu})_{4} \mathrm{H}_{2} & +\mathrm{HX} \longrightarrow \mathrm{M}_{3}(\mathrm{~N} / \mathrm{Bu})_{4} \mathrm{H}_{3} X \\
M & =\mathrm{Ge}, X=C \mid(1) ; x=B_{r}(2) \\
M & =S n, X=C \mid(4), X=B r(5)
\end{aligned}
$$

synthetic routes to these compounds are depicted in eqs 2 and 3.

$$
\begin{aligned}
& 2 \mathrm{Me}_{2} \mathrm{Si}(\mathrm{N} / \mathrm{BU})_{2} \mathrm{M}+\mathrm{Me}_{2} \mathrm{Si}(\mathrm{HN} / \mathrm{BU})(\mathrm{N} / \mathrm{BU}) \mathrm{MX}+ \\
& 4 \mathrm{~B} \cup \mathrm{NH}_{2} \\
& \longrightarrow \quad 3 \mathrm{Me}_{2} \mathrm{~S}(\mathrm{HN} / \mathrm{BU})_{2}+\mathrm{M}_{3}(\mathrm{~N} / \mathrm{BU})_{4} \mathrm{H}_{3} \mathrm{X} \\
& M=G e, X=C l(1) ; \\
& M=S \cap, X=C,(4), X=B,(5), X=\mid(6) \\
& 2 \mathrm{Me}_{2} \operatorname{si}(\mathrm{N} / \mathrm{Bu})_{2} \mathrm{M}+0.5[\mathrm{MX}(\mathrm{HN} / \mathrm{B} u)]_{2}+3 / \mathrm{BuNH} \\
& \longrightarrow 2 \mathrm{Me}_{2} \mathrm{Si}(\mathrm{HN} / \mathrm{Bu})_{2}+\mathrm{M}_{3}(\mathrm{~N} / \mathrm{Bu})_{4} \mathrm{H}_{3} \mathrm{X} \\
& M=G_{e}, X=C l(1): \\
& M=\sin X=0 \text { (1) } X=B,(5), X=1(0)
\end{aligned}
$$

These alternative methods require more easily obtainable starting materials, such as $\mathrm{Me}_{2} \mathrm{Si}(\mathrm{N}-t-\mathrm{Bu})_{2} \mathrm{M}$ and $\mathrm{Me}_{2} \mathrm{Si}(\mathrm{N}-t-\mathrm{Bu})(t-$ $\mathrm{BuNH}) \mathrm{MX}$, and avoid the tedious preparation of anhydrous $\mathrm{HI}$.

In eq 2 the cyclic bis(amino)germylene or -stannylene is treated with tert-butylamine in the presence of $\mathrm{Me}_{2} \mathrm{Si}(t-\mathrm{BuNH})(\mathrm{N}-t$ $\mathrm{Bu}) \mathrm{MX}(\mathrm{M}=\mathrm{Ge}, \mathrm{X}=\mathrm{Cl} ; \mathrm{M}=\mathrm{Sn}, \mathrm{X}=\mathrm{Cl}, \mathrm{Br}, \mathrm{I})$. In all cases complete aminolysis occurs with the metallacycle $\mathrm{Me}_{2} \mathrm{Si}(t$ $\mathrm{BuNH})(\mathrm{N}-t$-Bu) MX serving as the $\mathrm{HX}$ carrier. The only side product is the volatile silazane $\mathrm{Me}_{2} \mathrm{Si}(t-\mathrm{BuNH})_{2}$, which is easily removed from the mixture in vacuo. Equation 3 is very similar to eq 2 , the only exception being that the $\mathrm{HX}$ carrier now is $[(t-\mathrm{BuNH}) \mathrm{MX}]_{2}$. The fact that the product $[(t-\mathrm{BuNH}) \mathrm{SnI}]_{2}(7)$ is formed from aminolysis of $\mathrm{Me}_{2} \mathrm{Si}(t-\mathrm{BuNH})(\mathrm{N}-t-\mathrm{Bu}) \mathrm{MX}$ with $t$ - $\mathrm{BuNH}_{2}$, as depicted in eq 4 (in analogy to former procedures $),{ }^{13,14}$ is a strong indication that $[(t-\mathrm{BuNH}) \mathrm{MX}]$ is an intermediate in the formation of $1,4,5$, and 6 in eq 2 .

$$
\begin{aligned}
& 2 M e_{2} \operatorname{Si}(H N / B U)(N / B U) S n-1+2 / B U N H_{2} \\
& -2 M_{2} S i(H N / B U)_{2}+[/ B U N(H) S n I]_{2}
\end{aligned}
$$

The products $1,2,4,5$, and 6 are initially identified by their ${ }^{1} \mathrm{H}$ NMR spectra as two resonances in a ratio of 3:1. This spectral pattern is consistent with the molecules possessing $C_{3 v}(3 \mathrm{~m})$ symmetry in solution. The three equivalent tert-butylamino groups occur at low field $\left(\delta 1.43\right.$ for 1-3 and 1.41 for 4-6) as expected, ${ }^{12,15}$ while the resonance for the remaining tert-butylamino group (labeled $R^{\prime}$ in formula $A$ ) is found at higher field ( $\delta$ range 1.16-1.06; for specific values, see Table IV). This high-field shift indicates there is an inductive effect as the size of the anion 
Table III. Selected Bond Lengths $(\AA)$ and Angles (deg) (Some Averaged ${ }^{a}$ ) of Compounds $1,3-0.5 \mathrm{C}_{6} \mathrm{H}_{6}, 4-0.5 \mathrm{C}_{6} \mathrm{H}_{6}, 5$, and $\mathrm{Sn}_{3}(\mathrm{~N}-t-B u){ }_{4} \mathrm{H}_{2}{ }^{5}$ for Comparison

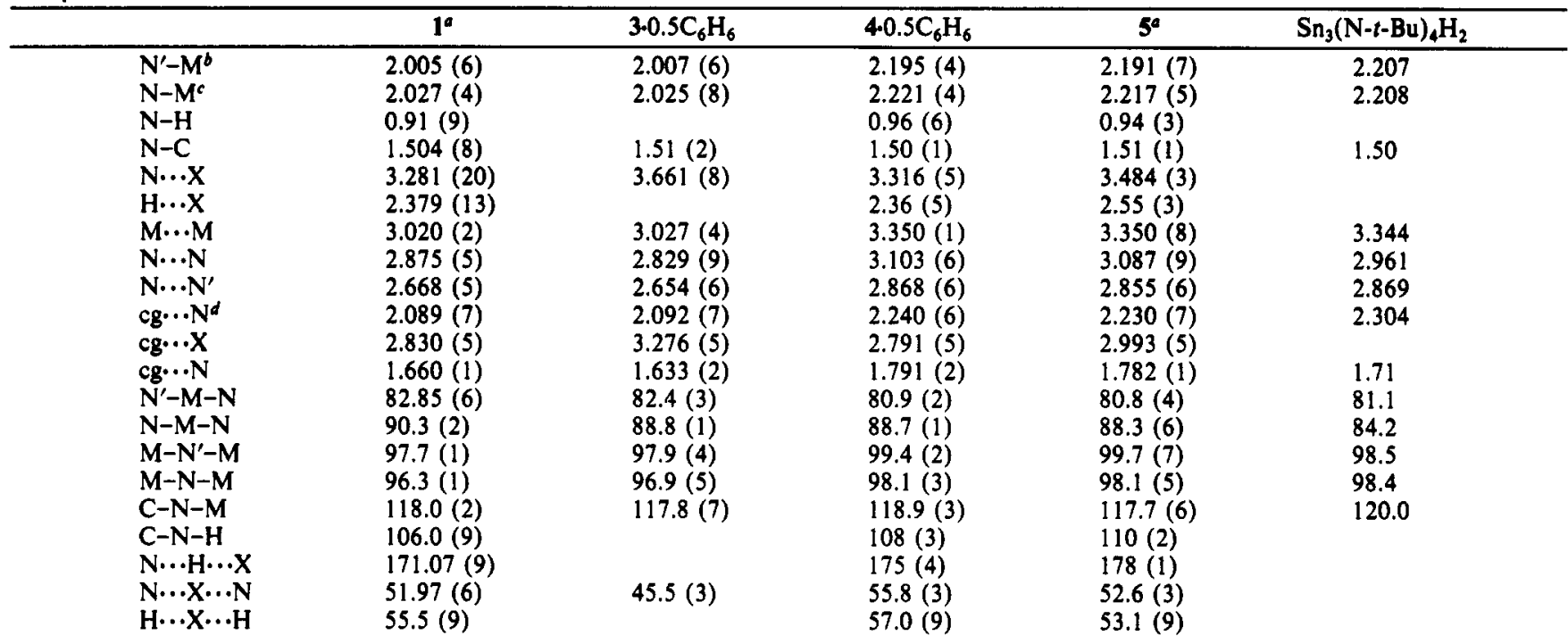

a Most of the distances and angles have been calculated from several chemically identical bonds. The mean deviation in parentheses is given here instead of the standard deviation, which may be smaller. ${ }^{6} \mathrm{~N}^{\prime}$ designates the $\mathrm{N}$ atom bonded to three metal atoms, which is situated on the (quasi) 3 -fold axis (compare formula $\mathrm{A}$ ). ${ }^{c} \mathrm{~N}$ designates the $\mathrm{N}$ atoms to which $\mathrm{H}$ atoms are bonded (compare formula $\mathrm{A}$ ). ${ }^{d} \mathrm{Cg}=$ center of gravity of the three hydrogen-linked $\mathbf{N}$ atoms.

Table IV. Physical and Spectroscopic Data for 1-6

\begin{tabular}{|c|c|c|c|c|}
\hline & $\mathrm{mp},{ }^{\circ} \mathrm{C}$ & ${ }^{1} \mathrm{H}$ NMR: $\delta$ & mass spectrum & $\begin{array}{c}\text { IR: } \\
\nu(\mathrm{NH}), \\
\mathrm{cm}^{-1}\end{array}$ \\
\hline 1 & $\begin{array}{l}150 \text { dec }^{\circ} \\
280 \text { dec }^{b}\end{array}$ & $\begin{array}{l}1.16(9 \mathrm{H}) \\
1.43(27 \mathrm{H})\end{array}$ & $\begin{array}{l}m / z=505 \\
\mathrm{C}_{16} \mathrm{H}_{39} \mathrm{~N}_{4}{ }^{72} \mathrm{Ge}_{2}{ }^{74} \mathrm{Ge}\end{array}$ & 3067 \\
\hline 2 & $\begin{array}{l}190 \text { deco }^{\circ} \\
280 \text { dec }^{b}\end{array}$ & $\begin{array}{l}1.13(9 \mathrm{H}) \\
1.43(27 \mathrm{H})\end{array}$ & $\mathrm{m} / \mathrm{z}=505$ & 3080 \\
\hline 3 & $\begin{array}{l}160 \mathrm{dec}^{\circ} \\
270 \mathrm{dec}^{b}\end{array}$ & $\begin{array}{l}1.11(9 \mathrm{H}) \\
1.43(27 \mathrm{H})\end{array}$ & $m / z=505$ & 3098 \\
\hline 4 & $289 \mathrm{dec}^{b}$ & $\begin{array}{l}1.11(9 \mathrm{H}) \\
1.41(27 \mathrm{H})\end{array}$ & $\begin{array}{l}m / z=643 \\
\mathrm{C}_{16} \mathrm{H}_{39} \mathrm{~N}_{4}{ }^{118} \mathrm{Sn}_{2}{ }^{120 \mathrm{Sn}}\end{array}$ & 3066 \\
\hline 5 & $290 \mathrm{dec}^{6}$ & $\begin{array}{l}1.08(9 \mathrm{H}) \\
1.41(27 \mathrm{H})\end{array}$ & $m / z=643$ & 3087 \\
\hline 6 & $\begin{array}{l}195 \operatorname{dec}^{a} \\
270 \operatorname{dec}^{b}\end{array}$ & $\begin{array}{l}1.06(9 \mathrm{H}) \\
1.41(27 \mathrm{H})\end{array}$ & $m / z=643$ & 3116 \\
\hline
\end{tabular}

increases from $\mathrm{Cl}^{-}$to $\mathrm{I}^{-}$. The compounds are only slightly soluble in noncoordinating solvents such as cyclohexane, benzene, or toluene, which may be taken as an indication of their polar bonds (see below). The compounds $1,2,4,5$, and 6 as well as 3 (for formation, see below) are furthermore characterized by mass and IR spectra and elemental analysis in addition to ${ }^{1} \mathrm{H}$ NMR spectra. Some of these data are collected for comparison in Table IV. The desorption chemical ionization $\left(\mathrm{DCI}^{+}\right)$mass spectra display parent ion envelopes centered at $m / z=505$ for 1-3 $(\mathrm{M}=\mathrm{Ge})$ and 642 for 4-6 $(M=S n)$ with characteristic isotope patterns for the calculated cations $\left[\mathrm{Ge}_{3}(\mathrm{~N}-t-\mathrm{Bu})_{4} \mathrm{H}_{3}\right]^{+}$and $\left[\mathrm{Sn}_{3}(\mathrm{~N}-t-\mathrm{Bu})_{4} \mathrm{H}_{3}\right]^{+}$, respectively. The repeated appearance of these ions and their stability within the mass spectrometer may be taken as a further hint at the cation/anion nature of these complexes. Another interesting feature is found in the $\nu(\mathrm{N}-\mathrm{H})$ wavenumbers of the compounds. There is an increase in these numbers, when $\mathrm{Cl}^{-}$is substituted by $\mathrm{Br}^{-}$or $\mathrm{I}^{-}$, respectively, for both the Ge series (1-3) and the Sn series (4-6). This fact seems to reflect the longer $H$...X distances, which result from the bigger halogens and which therefore should lead to less effective hydrogen bridging. In the structural part of this paper (see below), it can be shown that these wavenumbers are linearly correlated to the ionic radii of $\mathrm{Cl}, \mathrm{Br}$, and I or to the intramolecular distances obtained from the $\mathrm{X}$-ray structure determinations.

It seemed at this point evident that the molecules $1,2,4,5$, and 6 are composed of a spherical lipophilic part $\left(\mathrm{M}_{3}(\mathrm{~N}-t-\mathrm{Bu})_{4}\right)$ dominated by a quasitetrahedral arrangement of the tert-butyl groups and a lipophobic part $\left(\mathrm{H}_{3} \mathrm{X}\right)$ containing most of the polarity. Such species have directional behavior at the phase boundaries, with the "organic part" pointing into a noncoordinating organic solvent and the polar $\mathrm{H}_{3} \mathrm{X}$ arrangement pointing in the opposite direction. To test this assumption, we treated benzene solutions of 1 and 4 with the salts $\mathrm{NaBr}$ and $\mathrm{NaI}$ in heterogeneous reactions. As depicted in eqs 5 and 6,1 and 4 are transformed to $2,3,5$, and 6 , respectively, $\mathrm{NaCl}$ being the byproduct in all cases.

$$
\begin{aligned}
& \mathrm{M}_{3}(\mathrm{~N} / \mathrm{Bu}) \mathrm{H}_{3} \mathrm{Cl}+\mathrm{Nal} \longrightarrow \mathrm{M}_{3}(\mathrm{~N} / \mathrm{Bu})_{4} \mathrm{H}_{3} \mathrm{l}+\mathrm{NaCl} \\
& M=G_{e}(1) \quad M=G_{e}(3) \\
& M=S n(4) \quad M=S n(6)
\end{aligned}
$$

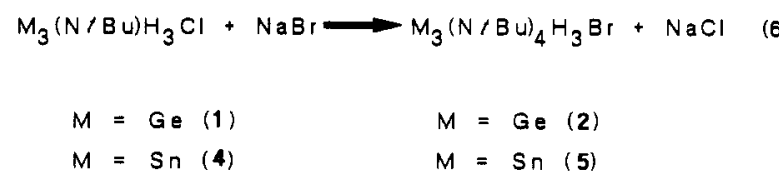

The iodine compound 3 is obtained here for the first time. Its data are compatible with those for the other homologous compounds (see Table IV). The yields for these reactions are almost quantitative although reaction time varies considerably. Sodium chloride can be identified by X-ray powder diffraction. This reaction is comparable to the transformation of $\mathrm{NaBr}$ to $\mathrm{NaCl}$ by interaction of gaseous $\mathrm{HCl}$ with solid $\mathrm{NaBr}{ }^{21}$ The transformation is ascribed to the higher polar nature and crystal lattice energy of $\mathrm{NaCl}$ compared to $\mathrm{NaBr}{ }^{22}$

If the crystal energy is a driving force in these reactions, we thought it should be possible to retransform 3 to 1 using a metal halide whose iodide is more stable than the chloride. As depicted in eq 7 , thallium chloride quantitatively transforms 3 back to 1 ,

$$
\mathrm{Ge}_{3}(\mathrm{~N} / \mathrm{Bu}) \mathrm{H}_{3} \mathrm{I}+\mathrm{TICl} \longrightarrow \mathrm{Ge}_{3}(\mathrm{~N} / \mathrm{Bu})_{4} \mathrm{H}_{3} \mathrm{Cl}+\mathrm{TII}
$$

with formation of TII. Here, as before, 1 is identified by ${ }^{1} \mathrm{H}$ NMR

(21) Harrison, L. G.; Siddiqui, R. A. Trans. Faraday Soc. 1962, 58, 982.

(22) Huheey, J. E. Anorganische Chemie; W. de Gruyter: Berlin, 1988; p 72. 


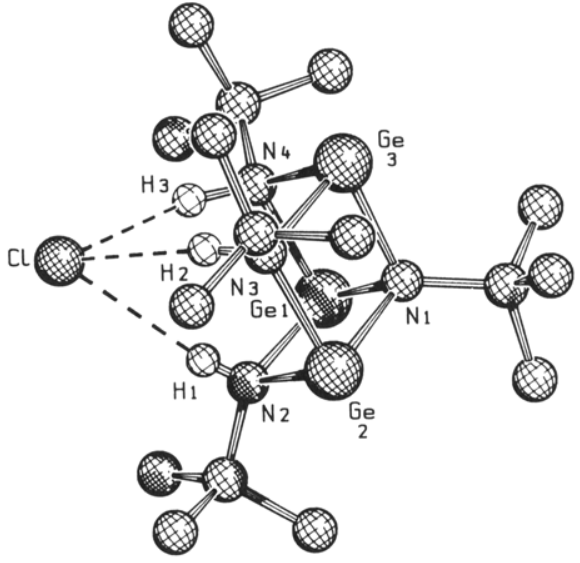

Figure 1. Graphic representation of $\mathrm{Ge}_{3}(\mathrm{~N}-t-\mathrm{Bu})_{4} \mathrm{H}_{3} \mathrm{Cl}$ (1) based on the single-crystal X-ray structure determination.

spectra and TII by its X-ray powder diffraction pattern.

The ratio of the reactants in eq 1 plays an important role. When an excess of $\mathrm{HX}$ was used, the cages react, forming products similar to those found in the $\mathrm{HCl}$ addition to $\mathrm{Sn}_{4}(\mathrm{~N}-t-\mathrm{Bu})_{4} \cdot{ }^{14}$ In eq 8 the products formed by subsequent addition of $\mathrm{HCl}$ to

$$
\begin{aligned}
& \mathrm{Sn}_{3}(\mathrm{~N} / \mathrm{Bu})_{4} \mathrm{H}_{2} \stackrel{\mathrm{HCl}}{\longrightarrow} \mathrm{Sn}_{3}(\mathrm{~N} / \mathrm{Bu})_{4} \mathrm{H}_{3} \mathrm{Cl} \\
& \stackrel{+5 \mathrm{HCl}}{-t \mathrm{BuNH}_{2}} 3 t \mathrm{BuNH}_{2} \cdot \mathrm{SnCl}_{2} \stackrel{+3 \mathrm{HCl}}{\longrightarrow} 3 / \mathrm{BuNH}_{3}^{+} \mathrm{SnCl}_{3}^{-}
\end{aligned}
$$

$\mathrm{Sn}_{3}(\mathrm{~N}-t-\mathrm{Bu})_{4} \mathrm{H}_{2}$ are summarized. The $\mathrm{HCl}$ adduct of $\mathrm{Sn}_{3}(\mathrm{~N}-t-$ $\mathrm{Bu})_{4} \mathrm{H}_{2}(4)$ is formed first. Further addition of $\mathrm{HCl}\left(\mathrm{Sn}_{3}(\mathrm{~N}-t\right.$ Bu) ${ }_{4} \mathrm{H}_{2}: \mathrm{HCl}=1: 6$ ) destroys the cage 4 to form $t-\mathrm{Bu}-\mathrm{NH}_{2} \cdot \mathrm{SnCl}_{2}$ and finally $t-\mathrm{BuNH}_{3}{ }^{+} \mathrm{SnCl}_{3}{ }^{-}\left(\mathrm{Sn}_{3}(\mathrm{~N}-t-\mathrm{Bu})_{4} \mathrm{H}_{2}: \mathrm{HCl}=1: 9\right)$. The four-membered ring $(t-\mathrm{BuN}(\mathrm{H}) \mathrm{SnCl})_{2}$ has not been found to be stable under these conditions, in contrast to the reaction of $\mathrm{Sn}_{4}(\mathrm{~N}-t-\mathrm{Bu})_{4}$ with $\mathrm{HCl} .^{14}$

Structural Details. $\mathrm{X}$-ray structural determinations have been carried out on single crystals of $1,3 \cdot 0.5 \mathrm{C}_{6} \mathrm{H}_{6}, 4 \cdot 0.5 \mathrm{C}_{6} \mathrm{H}_{6}$, and 5 . While $3 \cdot 0.5 \mathrm{C}_{6} \mathrm{H}_{6}$ and $\mathbf{4} \cdot 0.5 \mathrm{C}_{6} \mathrm{H}_{6}$ are isotypical, crystallizing in the rhombohedral space group $R \overline{3}, 1$ crystallizes in a monoclinic $\left(P 2_{1} / n\right)$ and 5 in an orthorhombic (Pnma) space group. The crystals of 1 and 5 contain no interstitial benzene and form closed-packed (1) or mostly closed-packed (5) van der Waals lattices. ${ }^{23} \mathbf{1}$ has no crystallographic symmetry, whereas $\mathbf{5}$ is situated on a mirror plane of the space group. A closer look at the bond lengths and angles, however, reveals there is only a minor deviation from the higher $C_{3 v}(3 \mathrm{~m})$ symmetry. The molecules thus lose the higher symmetry, which exists in solution, as they are packed in the crystal lattice following the principles formulated by Kitaigorodskii. ${ }^{23}$

In Figure 1 the compound $\mathrm{Ge}_{3}(\mathrm{~N}-t-\mathrm{Bu})_{4} \mathrm{H}_{3} \mathrm{Cl}(1)$ is shown to illustrate the general overall structure of the molecules. Figure 2 gives a view down the 3 -fold axis of $\mathrm{Sn}_{3}(\mathrm{~N}-t-\mathrm{Bu})_{4} \mathrm{H}_{3} \mathrm{Cl}(4)$, showing the high symmetry of the molecules. As can be seen from these representations, the central cage of the molecules $\mathrm{M}_{3}(\mathrm{~N}$ $t$-Bu ${ }_{4} \mathrm{H}_{3} \mathrm{X}$ can be described as a $\mathrm{M}_{3} \mathrm{~N}_{4} \mathrm{X}$ "cube". Here the corner $\mathrm{X}$ is displaced from the center but held by three hydrogen atoms bridged to the three nitrogen atoms. The same cage may also be described as an $\mathrm{N}_{4}$ tetrahedron with three faces pyramidally capped by the metallic elements and one face capped by a $\mathrm{H}_{3} \mathrm{X}$ trigonal pyramid.

In the rhombohedral system the molecules 3 and $\mathbf{4}$ adopt the relatively high point symmetry $C_{3}(3)$, losing only the mirror plane. Figure 3 shows a section of the packing of $4 \cdot 0.5 \mathrm{C}_{6} \mathrm{H}_{6}$. When viewed down the 3 -fold axis of the lattice, "pairs of molecules" are separated by benzene molecules, which are situated around $\overline{3}$ centers. These benzene molecules are in between two tert-butyl

(23) Kitaigorodskii, A. I. Molecular Crystals and Molecules; Academic Press: New York, 1973.

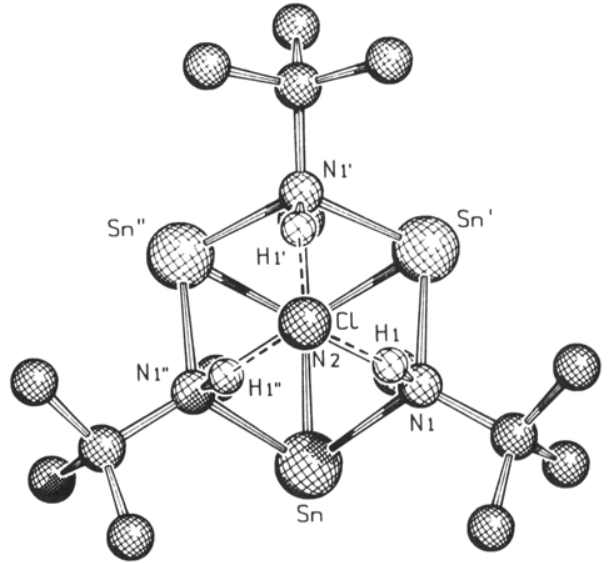

Figure 2. View down the 3-fold axis of $\mathrm{Sn}_{3}(\mathrm{~N}-t-\mathrm{Bu})_{4} \mathrm{H}_{3} \mathrm{Cl}$ (4) with $\mathrm{Cl}$ as the nearest atom.

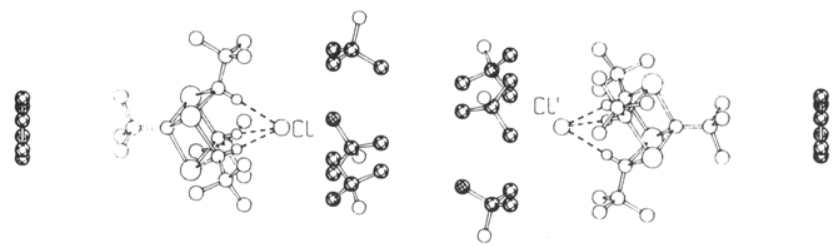

Figure 3. Section of the packing of 4 to show the interstitial benzene molecules (left and right). The tert-butyl amino groups in the middle of the picture belong to six different molecules, which are omitted for clarity.

groups of different molecules. Interestingly, these "pairs" have opposing halogens $(3, \mathrm{I} \cdots \mathrm{I}=11.34 \AA ; 4, \mathrm{Cl} \cdots \mathrm{Cl}=12.31 \AA)$ at very long distances. The long separation of the halogen atoms of the molecule pair is caused by tert-butyl groups of other molecules (Figure 3).

It is interesting to note that $\mathbf{1}$, as well as $\mathbf{5}$ and $\mathbf{6}$, when crystallized from benzene, form crystals isotypical to $3 \cdot 0.5 \mathrm{C}_{6} \mathrm{H}_{6}$ and 4.0.5 $\mathrm{C}_{6} \mathrm{H}_{6}$ (rhombohedral lattices: $1 \cdot 0.5 \mathrm{C}_{6} \mathrm{H}_{6}, a=b=11.495$ (7) $\AA, c=34.54$ (2) $\AA, V=3953 \AA^{3} ; 5 \cdot 0.5 \mathrm{C}_{6} \mathrm{H}_{6}, a=b=11.807$ (6) $\AA, c=35.27$ (2) $\AA, V=4258 \AA^{3} ; 6 \cdot 0.5 \mathrm{C}_{6} \mathrm{H}_{6}, a=b=11.996$ (5) $\AA, c=35.53$ (2) $\AA, V=4428 \AA^{3}$ ).

At a first view, the bond lengths of $\mathbf{4}$ and $\mathbf{5}$ are only slightly different from those in $\mathrm{Sn}_{3}(\mathrm{~N}-t-\mathrm{Bu})_{4} \mathrm{H}_{2}$ and, generally speaking, the $\mathrm{M}_{3} \mathrm{~N}_{4} \mathrm{H}_{3} \mathrm{X}$ cages, when germanium is a compotent, are smaller than those where $\mathrm{M}=\mathrm{Sn}$. A closer look reveals nevertheless that important changes are taking place, if a molecule of $\mathrm{HX}$ is added to the neutral $\mathrm{Sn}_{3}(\mathrm{~N}-t-\mathrm{Bu})_{4} \mathrm{H}_{2}$ moiety: One can distinguish clearly between two kinds of metal-nitrogen bonds, the bonds between the nitrogen on the 3-fold axis and the metal atoms being shorter than these between the hydrogen-bearing nitrogen and metal atoms. While the nonbonding distances $\mathrm{N}$... $\mathrm{N}^{\prime}$ (for labeling compare formula A) in $\mathbf{4}$ and $\mathbf{5}$ differ only slightly compared to those in the neutral molecule $\mathrm{Sn}_{3}(\mathrm{~N}-t-\mathrm{Bu})_{4} \mathrm{H}_{2}$, the $\mathrm{N}$... N distances are much longer. The incorporation of $\mathrm{HX}$ in the polycycle seems to occur at the expense of the $\mathrm{M}-\mathrm{N}$ bonding and leads to an "opening" of the hole in the $\mathrm{M}_{3} \mathrm{~N}_{4}$ cage (compare also N-M-N angles with $\mathrm{N}-\mathrm{M}-\mathrm{N}^{\prime}$ ). This place is needed to accommodate the third hydrogen atom, which, of course, should have a repulsive force toward the other two hydrogen atoms already present. In $1,4 \cdot 0.5 \mathrm{C}_{6} \mathrm{H}_{6}$, and 5 the positions of the hydrogen atoms bonded to nitrogen can be located. They have in the three structures similar intramolecular distances $(\mathrm{H} \cdots \mathrm{H}=2.23$ (1), 2.23 (4) $\left.\left.0.5 \mathrm{C}_{6} \mathrm{H}_{6}\right), 2.27 \AA(5)\right)$. Despite the different compositions of the compounds the interatomic $\mathrm{H} \cdot . . \mathrm{H}$ distances in $\mathbf{1}$ are equal to those found in $4 \cdot 0.5 \mathrm{C}_{6} \mathrm{H}_{6}$ !

The halides in these compounds are clearly pyramidally coordinated by three hydrogen atoms. As far as determined, the $\mathrm{H} \cdot \ldots \mathrm{X} \cdot . \mathrm{H}$ angles are quite accute and are somewhat greater than the $\mathrm{N}$... $\mathrm{X}$... N angles. This implies that the hydrogen bridges are situated somewhat outside the $\mathrm{N}_{3} \mathrm{X}$ pyramid although the $\mathrm{N}$... $\mathrm{H}$... X angles are very close to $180^{\circ}$ (compare Table III). The 


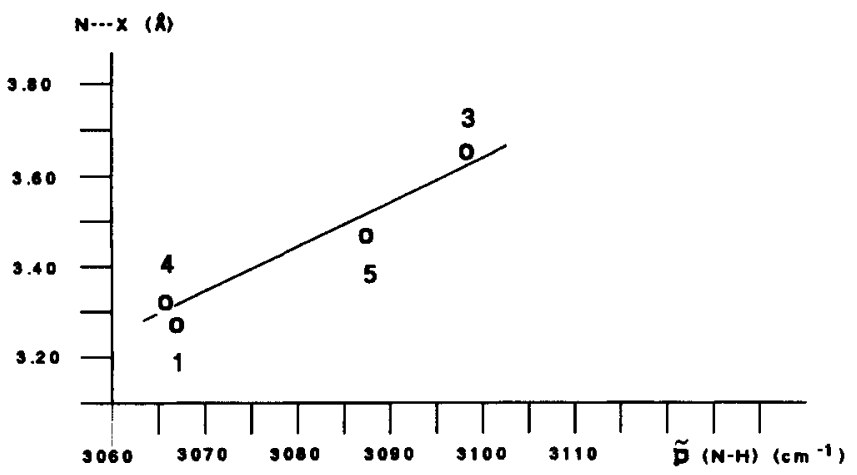

Figure 4. No..X distances as a function of the $\nu(\mathrm{N}-\mathrm{H})$ wavenumbers.

nearly linear $N$...H...X arrangements suggest relatively strong bridges. This becomes evident, if the $\mathrm{N}$... $\mathrm{X}$ distances found here are compared to the CsCl-type structures (disorder of the $\mathrm{NH}_{4}^{+}$ ions) of ammonium chloride $(\mathrm{N} . . \mathrm{Cl}=3.356 \mathrm{~A})$, ammonium bromide $(\mathrm{N} \ldots \mathrm{Br}=3.507 \AA)$, and ammonium iodide $(\mathrm{N} \ldots \mathrm{I}=3.737$ A). ${ }^{24,25}$ Contrarily the $\mathrm{N}$... Cl distances in $\mathrm{CH}_{3} \mathrm{NH}_{3} \mathrm{Cl}(\mathrm{CN}$ at $\mathrm{Cl}=4)$ are $3.18 \AA$, those in $\left(\mathrm{CH}_{3}\right)_{2} \mathrm{NH}_{2} \mathrm{Cl}(\mathrm{CN}$ at $\mathrm{Cl}=2)$ are $3.11 \AA$, and those in $\left(\mathrm{CH}_{3}\right)_{3} \mathrm{NHCl}(\mathrm{CN}$ at $\mathrm{Cl}=1)$ are $3.005 \AA$, respectively, and therefore considerably shorter than those in 1 and $4 .{ }^{26}$ Compared to the complexes formed by $\mathrm{Cl}^{-}$and $\mathrm{Br}^{-}$with cryptands of a different charge, ${ }^{7,9,10 b}$ the $\mathrm{N}$... $\mathrm{X}$ distances found in 1,4 , and 5 are again too long. A typical value for four-coordinate chloride $\mathrm{N} \ldots \mathrm{Cl}$ is 3.09 (2) $\AA$, and for six-coordinate chloride and bromide $\mathrm{N} \ldots \mathrm{Cl} / \mathrm{Br}$ values are around $3.30(9)$ and 3.39 (4) $\AA$, respectively. This discrepancy cannot be explained by the different numbers of hydrogen atoms, which coordinate the chloride ( 3 versus $6,4,2$, or 1 ). The compounds 1 and 4 constitute a special case (for explanation see below).

The $\mathrm{N}-\mathrm{H}$ bond lengths (compare Table III) are routinely determined too short in X-ray analyses and should be near 1.02 $\AA$ (cf. the literature ${ }^{25}$ ). They nevertheless indicate that the $\mathrm{N} \ldots \mathrm{H} \cdots \mathrm{X}$ bridge is highly unsymmetrical, the hydrogen atom being principally bonded to nitrogen. In summary, it seems to be clear

(24) Jones, K. The Chemistry of Nitrogen. In Comprehensive Inorganic Chemistry; Pergamon Press: Oxford, England, 1975; Chapter 19, p 245.

(25) Parsonage, N. G.; Staveley, L. A. Disorder in Crystals; Clarendon Press: Oxford, England, 1978.

(26) Lindgren, J.; Olovson, I. Acta Crystallogr. 1968, B24, 554. from the geometric arrangements of $\mathrm{H}$ and $\mathrm{X}$ atoms that an attractive force must be assumed between hydrogen and the halogen atoms, which is presumably of the ionic type with a strong directional character. The No..X distances correlate well with the $\nu(\mathrm{N}-\mathrm{H})$ wavenumbers, as may be seen from Figure 4.

At this stage a comparison of the molecules 1 and 4 may improve the understanding of the bonding. As can be seen in Table III, the N...Cl distances in 1 are shorter than those in 4 , but the distance from the center of gravity $(\mathrm{cg})$ of the three hydrogenbonded nitrogen atoms to $\mathrm{Cl}$ is longer in 1 than in 4 . Clearly, the $\mathrm{Sn}_{3} \mathrm{~N}_{4}$ polycycle is better suited to accommodate a chloride or even a bromide than the $\mathrm{Ge}_{3} \mathrm{~N}_{4}$ polycycle. This is also reflected in the $N \cdots X \ldots N$ angles, which increase in the order $3<1<5$ $<4$, the germanium compound 1 interestingly displaying a smaller angle than the tin compounds 4 and even 5 . To use another picture, the halogen atoms are drawn further into the tin cage than into the germanium cage (see also distances cg... $\mathrm{N}$ in Table III), as the hole in the tin cage is deeper (cg...X distances) and larger ( $N$... $N$ or $N$...X distances) than that of the germanium analogue. The astonishingly long $N$... $X$ distances found in $\mathbf{1}$ and 4 , compared to the methylammonium chlorides or to cryptates (see before), must be attributed to the fact that the amino-hydrogen atoms are close together (see above). Besides attractive forces between $\mathrm{Cl}$ and $\mathrm{H}$ repulsive forces between $\mathrm{Cl}$ and the three nitrogen atoms, to which hydrogen is bonded, must be considered.

\section{Conclusion}

The physical, spectral ( $\delta$ ('H NMR) $\nu$ (IR)), and crystallographic data of 1-6 are in agreement with a description as molecular complexes with a high degree of "zwitter ionic" character between the $\mathrm{M}_{3}(\mathrm{~N}-t-\mathrm{Bu})_{4} \mathrm{H}_{3}{ }^{+}$and the $\mathrm{X}^{-}$part of the cage molecule. The exchange of the halogen atoms in biphasic reactions can be attributed to the special geometry and the polar structures of the compounds as well as to the bonding. The possible use of 1-6 as phase-transfer catalysts seems evident. Further experiments concerning the reactivity of these molecules are under investigation.

Acknowledgment. Financial support from the Fonds der Chemischen Industrie and the DFG is gratefully acknowledged. T.R.P. thanks the University of the Saarland for financial support. We thank Dr. L. Stahl for reading the manuscript.

Supplementary Material Available: Tables giving crystallographic details, thermal parameters, and bond distances and angles (10 pages); listings of structure factors ( 37 pages). Ordering information is given on any current masthead page. 